\title{
Time, Imaginary Value, Paradox, Sign and Space
}

\author{
Louis H. Kauffman \\ Math, UIC \\ 851 South Morgan Street \\ Chicago, IL 60607-7045 \\ <kauffman@uic.edu>
}

"Fire rests by changing." -- Heraclitus

\begin{abstract}
This paper discusses paradox and imaginary values in relation to the mutuality of sign and space.
\end{abstract}
Key Words: time, imaginary, paradox, sign, space.

\section{INTRODUCTION}

We will discuss the role of time and imagination in the unfolding of circular processes and the relation of these concepts to "imaginary" values in logic and mathematics. In particular, we will discuss the Flagg Resolution of paradoxes and its use of algebraic simultaneity. These mathematical issues will then be considered from the point of view of semiotics in the sense of C. S. Peirce. We wish to explore the relationship of this way of holding Signs with the mathematics. This discussion will include an examination of how a shift in viewpoint can transform a spatial context to a spacetime context. Along with examples from linguistic and social contexts, this includes the transformation of spatial relations to processes in spacetime that underlie the structure of Feynman diagrams and topological quantum field theory. This paper is based on my columns on Virtual Logic [35] and on a recent paper of mine on the mathematics of Charles Sanders Peirce [42].

\section{A CLASSICAL PARADOX IN LOGIC - EPIMENIDES' LIAR PARADOX}

Consider the statement:

$$
\mathbf{J}=\text { "This statement is false." }
$$

If $\mathrm{J}$ is true then $\mathrm{J}$ states that $\mathrm{J}$ is false.

Thus we can write

$$
\text { If } \mathrm{J} \text { is false then } \mathrm{J} \text { states that } \mathrm{J} \text { is true. }
$$

$$
\mathbf{J}=\text { Not } \mathbf{J} \text {. }
$$

One might shun such a statement, but perhaps there is a way to deal with it. One solution that has been proposed is to say that $\mathrm{J}$ has a new kind of truth value, neither true nor false, and to devise a logic with more than two truth values in which the statement $\mathrm{J}$ can live. In this 3 (or more)-valued logic some rules of standard logic seem to demand sacrifice. It has traditionally been observed that the paradox would disappear if we dropped the rule

$$
\text { True }=P \text { or Not } P
$$

and made a new logic that did not assume this "law of the excluded middle." 
This approach is quite interesting and it leads to whole new fields of logic. Nevertheless, it is possible that the law of the excluded middle is not the only rule we could drop that would keep the contradiction from occurring, and perhaps nothing need be really lost!

\section{THE FLAGG RESOLUTION}

Around 1980 I had the good fortune to meet James Flagg who told me of his original way to resolve the Liar Paradox and other paradoxes in mathematics and logic.

Flagg observes that the sentence

$$
\mathbf{S}=\mathbf{J} \text { or Not } \mathbf{J} \quad \text { (with } \mathbf{J}=\text { Not } \mathbf{J}) \text {. }
$$

would not give us all this trouble if we took to heart the following rule:

THE FLAGG RESOLUTION

YOU ARE NOT ALLOWED TO EXCHANGE "J" FOR "NOT J" ANYWHERE UNLESS YOU DO SO EVERYWHERE. THERE IS ONE AND ONLY ONE EXPRESSION "J". SUBSTITUTION OF "NOT J" FOR "J" (OR VICE VERSA) IS THEREFORE A GLOBAL REPLACEMENT. OTHER THAN THIS SPECIAL TREATMENT OF "J", NO RULES OF LOGIC ARE CHANGED FROM CLASSICAL LOGIC.

That is, if you wish to make a substitution of "J" for "Not $\mathrm{J}$ " the substitution must be done for every appearance of "J".

Lets see how the Flagg Resolution applies to our particular paradox of the previous section.

True $=\mathbf{J}$ or Not $\mathbf{J}$

If, in this expression, we wish to replace $\mathrm{J}$ by Not $\mathrm{J}$ we can do it to both $\mathrm{J}$ 's and conclude

True $=$ Not $\mathbf{J}$ or Not Not $\mathbf{J}$.

We can write

\section{Not $\operatorname{Not} \mathbf{J}=\mathbf{J}$}

since this is a rule of ordinary logic and finally conclude

True $=$ Not $\mathbf{J}$ or $\mathbf{J}$.

This is the same as

$$
\text { True }=\mathbf{J} \text { or Not } \mathbf{J}
$$

since $(\mathrm{A}$ or $\mathrm{B})$ and $(\mathrm{B}$ or $\mathrm{A})$ are equivalent in ordinary logic.

No paradox appears.

The beauty of the Flagg Resolution is that we can accommodate new logical values and self-reference (like J) into the reasoning process without making artificial adjustments to the logic itself.

Historical Remark. The Resolution originally came about as the result of a conflict in point-of-view between F. J. Varela [40] and G. Spencer-Brown [9]. Varela wrote a paper [40] showing how to replace the Calculus of Indications of G. Spencer-Brown (see previous columns in this series) with a "Calculus for Self-Reference" that embodied the patterns of three-valued logic with the third value interpreted as autonomy or self-reference. This constituted Kleene's three-valued logic interpreted for self-reference. (See [38] for the metamathematical point of view.) The Varela calculus is excellent, but not necessary to patch any paradox in Spencer-Brown. This is the lesson of the Flagg Resolution. For a discussion of the relationship of the Varela calculus with the Flagg Resolution, see my paper [16] "Imaginary Values in Mathematical Logic". The Flagg Resolution put the very beautiful Calculus of Indications back on its stated ground - contrary to Varela. In the course of that discussion, the Resolution was discovered to have wider applications. 
The reader will by now have appreciated that the Flagg Resolution raises numerous questions about logical and mathematical practice. Is there a precedent for this treatment of paradox? Will it apply to apparently deeper paradoxes such as the Russell paradox? What is the meaning of this global nature of the entity J? If J is an exemplar of a "cybernetic unity", what is the meaning of this global nature?

\section{WAVEFORMS AND TIME SERIES}

There is a model for the Flagg Resolution that is based on a model of temporality. Think of $\mathrm{J}$ as a temporal sequence or alternation of True $(\mathrm{T})$ and False $(\mathrm{F})$ :

$$
\mathrm{J}=\ldots \ldots \text {.... TFTFTFTFTFTFTFTFTFTFTFTFTFTFTFTF } \ldots \ldots \ldots
$$

You can also think of such a sequence as a time series

$$
\mathbf{J}\{\mathbf{t}\}(=\mathbf{T} \text { or } \mathbf{F})
$$

where $t$ is any integer (we proceed from time in the infinite past to time in the infinite future). The series Not $\mathbf{J}$ is defined by the equation

$$
(\operatorname{Not} J)\{t\}=\operatorname{Not} J\{t\}
$$

so that Not $\mathrm{J}$ is false exactly when $\mathrm{J}$ is true and vice versa.

If you look at $\mathrm{J}$ and Not $\mathrm{J}$ together at any given time then certainly

$$
\text { J or Not } \mathbf{J}=\mathbf{T}
$$

because one or the other of them is true at any given time.

On the other hand, if you look at $\mathbf{J}$ alone or Not $\mathbf{J}$ alone as patterns then you are tempted to identify them since they are just shifted by one time step:

$$
\begin{aligned}
\mathbf{J} & =\ldots \ldots \text { TFTFTFTFTFTFTFTFTFTFTFTFTFTFTFTF } \ldots \ldots \ldots \\
\text { Not } \mathbf{J} & =\ldots \ldots
\end{aligned}
$$

Thus in this model, the statement $\mathbf{J}=$ Not $\mathbf{J}$ becomes a meta-statement about the structure of the time series $\mathbf{J}$, while the Flagg Resolution's law of substitution becomes a way of maintaining synchronicity by making either no phase shift, or simultaneous phase shifts in the course of calculation. In this model it would be ludicrous to consider $\mathbf{J}$ or Not $\mathbf{J}$ as reducible to $\mathbf{J}$ !

Instead of the time series we can model $\mathbf{J}$ as an ordered pair

$$
\mathbf{J}=[\mathbf{T}, \mathbf{F}]
$$

where this constitutes two steps in the order of the time series. Then

$$
\text { Not } \mathbf{J}=[\mathbf{F}, \mathbf{T}]
$$

if we regard the negation of the series as the new series obtained by negating each of its temporal values, and

$$
\mathbf{J}=\mathbf{N o t} \mathbf{J}
$$

only in the sense of confusing the order of these two-step sequences.

In fact it was exactly because of this interpretation of extra-logical values in terms of waveforms that Flagg and I immediately realized our rapport on this issue. I had, when Flagg and I first met, just finished writing some papers on a waveform interpretation where the context was a four valued logic that did not allow the law of the excluded middle! (See [16], [12], [37], [14].) 
What I had done was say "Let us change the definition of Not so that Not $\mathbf{J}=\mathbf{J}$ and let us retain the time series." This is done by saying that the "Not" of a time series includes negating every term and shifting the whole series by one step! This is a really trenchant idea. It means that one is including a delay of one time step in the operation of negation. In this model we have

Then

$\operatorname{Not}[A, B]=[\operatorname{Not} B, \operatorname{Not} A]$.

I call

$$
\operatorname{Not}[T, F]=[\operatorname{Not} F, \operatorname{Not} T]=[T, F] \text {. }
$$

and

$$
\mathbf{I}=[\mathbf{F}, \mathbf{T}]
$$

$$
\mathbf{J}=[\mathbf{T}, \mathbf{F}] \text {. }
$$

I have two distinct wave forms I and J, each satisfying its own negation, each looking like the other in isolation, and together we have

$$
\mathbf{I J}=\mathbf{T} \text {. }
$$

In the formalism of the ordered pairs this is expressed as

$$
[A, B] \text { or }[C, D]=[A \text { or } C, B \text { or } D] \text {. }
$$

and $\mathrm{T}$ is identified with the pair [T,T] while $\mathrm{F}$ is identified with the pair [F,F].

Remember that the ordered pairs stand for time series and that $[\mathbf{T}, \mathbf{T}]$ just stands for the series

TTTTTTTTTTTTTTTTTTTT

that states True at all times.

In my waveform model for four valued logic I had everything nicely in place and now you can see what happened to the law of the excluded middle! In my model it was just plain that

was simply not equal to $\mathrm{T}$.

\section{I or Not I}

An exposition of the waveform algebra and corresponding dynamics of forms can also be found in Chapter 12 of Varela's book "Principles of Biological Autonomy" [10]. This chapter is an essentially verbatim rendering of our [Kauffman and Varela] joint paper "Form Dynamics" [12].

Multiple valued logics are ways to encapsulate dilemmas in controlled contexts that admit calculations. In mathematics there are many parallels to this. You can see however, that it was not necessary to do anything as drastic as the four-valued logic to accommodate waveforms. The Flagg Resolution provides the abstract background for an atemporal view of this temporal resolution.

G. Spencer-Brown in "Laws of Form" [9] Chapter 11 said

"Since we do not wish, if we can avoid it, to leave the form, the state we envisage is not in space but in time.".

Originally one would have interpreted this transition into time as directly arising from the recursive series implicit in any given re-entering or self-referential form (such as $\mathbf{J}=\mathbf{N o t} \mathbf{J}$ ). In other words a paradox creates a buzzer when you implement it (off implies on and on implies off); yet the implemented circuit is a process and is therefore not a paradox. With the Flagg Resolution we see that the modification of a simple law of substitution in algebraic logic is sufficient to create a formal image of simultaneity. This simultaneity is not in a state of time (or space). It is the precursor, or archetype of timespace.

\subsection{And Recursion Itself}

The implicit nature of recursions is well known to those who study them. In a single equation lives a whole complex world. Perhaps the most famous example is the Mandelbrot set, defined through the equation $f(z)=z^{2}+k$ where $\mathrm{k}=(\mathrm{a}+\mathrm{bi})$ is a complex number. Depending upon the value of $\mathrm{k}$, the iteration

$$
f(f(f(\ldots f(0) \ldots)))=f^{(n)}(0)
$$


either does or does not go to infinity as n goes to infinity. The Mandelbrot set is the collection of all $\mathrm{k}$ where the iteration does not approach infinity. It is a deep and complex structure implicit in a small equation.

There are other examples of this phenomenon of order implicate in recursion that are directly related to the discussion in this section.

For example, one can consider $\mathrm{f}(\mathrm{x})=\mathrm{a}+\mathrm{b} / \mathrm{x}$ and its iteration producing the continued fraction $\mathrm{f}(\mathrm{f}(\mathrm{f}(\ldots)))$ :

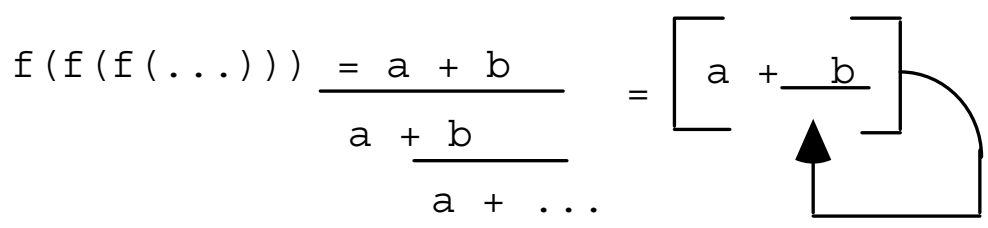

We envisage the recursive form

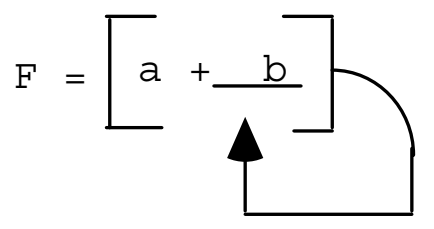

as representing a "value" such that $\mathrm{F}=\mathrm{a}+\mathrm{b} / \mathrm{F}$. Thus $\mathrm{F}$ contains all of the implicate structure of the recursion associated with $f$. We can ask in what domain $F$ might live? If $F=a+b / F$, then assuming $F$ has numerical properties, we have $F^{2}=a F+b$. This is a quadratic equation and has roots $\left(a+\sqrt{ }\left(a^{2}+4 b\right)\right) / 2$ and $\left(a-\sqrt{ }\left(a^{2}+4 b\right)\right) / 2$. Assuming that $a$ and $b$ are positive, it is a fact that when the roots are real $\left(a^{2}+4 b>=0\right)$ then the continued fraction $F$ converges to $\left(a+\sqrt{ }\left(a^{2}+4 b\right)\right) / 2$. For example

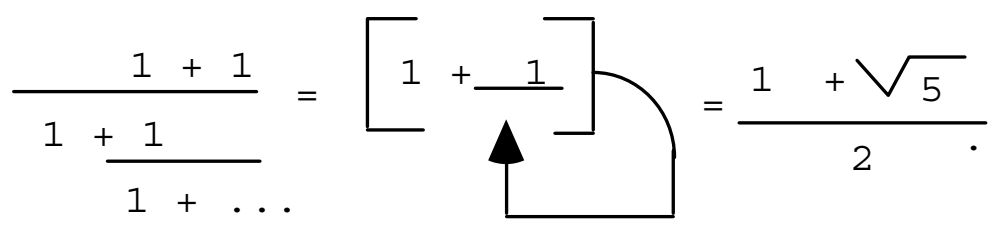

However, if $a^{2}+4 b<0$, then there is no real value for this continued fraction, and one may say that its value corresponds to the complex number roots of the quadratic equation, but then this requires thought and comparison to make sense out of that metaphor. In the case of $a=0$ and $b=-1$ we have $f(x)=-1 / x$ with complex roots $i$ and $-i$ with $\mathrm{i}^{2}=-1$. Thus we can assert, within this metaphor that
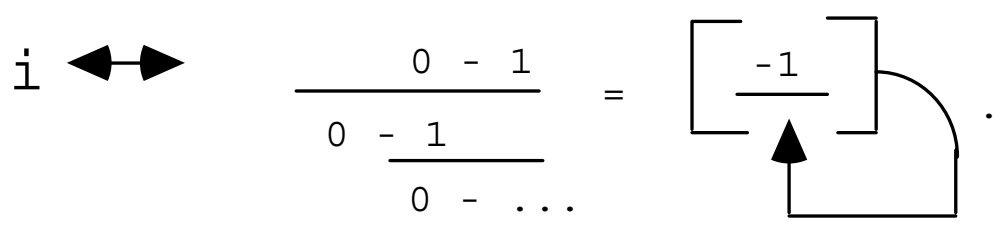

This part of the discussion is essentially the same as our discussion of the paradox $R=\sim R$ but transposed to the realm of numbers where the oscillation between True and False has been replaced by an oscillation between +1 and -1 . When we go further and take other quadratics with complex roots such as $f(x)=1-2 / x$ beautiful chaotic phenomena occur in the iteration and indeed these can be explained in terms of the complex numbers (See [43].) The deep dialogue among numbers, imaginary numbers and recursive forms shows clearly that what we regard as classical mathematics can be seen as an imaginary domain in which paradox resolves into the patterns of iterative process.

I cannot leave this section without mentioning one more recursion. It is the process equation [44]

$$
A(t+1)=A(t)+g \sin ((A(t))
$$

Here there is a wealth of phenomena generated through elemental bipolar feedback. When the coupling constant $\mathrm{g}$ is 
small, then the iteration is periodic, then there is a transition to chaos and after chaos (around $g=4.7$ ) to what we have called bios, a times series partaking of chaos and yet resembling the patterns of biological time.

Incredible worlds come into being beyond the dichotomy of True and False.

\section{MATHEMATICS, STRUCTURE AND TIME}

Sometimes I think that Mathematics is an enterprise self-designed to do away with time. No matter that you started with a temporal process or a time series, by the time (sic) you have formulated your data and the problem in mathematical terms, time has disappeared into structure. The essential quality of time that opens up a future is not formalized in mathematics and yet it is lingering there in the surprises of a recursion and the unimagined consequences of a given set of assumptions.

In the Flagg Resolution we give a structural linkage between $\mathrm{J}$ and Not $\mathrm{J}$ that corresponds or is isomorphic to their relative phase shifts in a temporal interpretation. It is certainly not necessary to take this interpretation. Without the interpretation, we have an entity whose structural self-description ( $J=$ Not $J$ ) is linked with its appearance in the formal text of the mathematics. (Hence it can not be exchanged locally for its negation.)

A text of Mathematics obeying the rule of substitution inherent in the Flagg Resolution is no longer an objective, or classical description. This text is dependent upon the relative positioning of functions of $\mathrm{J}$ within that text. The values of the text are functions of its own structure. There is no separating the text and what that text describes.

The statement (a small text)

$$
\mathbf{S}=\mathbf{J} \text { or Not } \mathbf{J}
$$

has value True not through the description of an external state of affairs about $\mathbf{J}$ (We have abandoned the temporal interpretation for this discussion.), but through the extension of a known logical relation beyond its normal call of duty. From the truth of "J or Not J", I cannot deduce that either $\mathrm{J}$ is true or that Not $\mathrm{J}$ is true. These factors, like strange puzzle pieces are neither True nor False and yet, in combination, a definite truth value emerges.

It is here that we reach the essential circularity and inseparability of the issue, the cybernetic hub of the matter. The ground of our reason is neither true nor false, but structural and global. In this ground the system and its observer are neither separate, nor coincident. Taken singly, system and observer have no logical values, nor any determinate form. Taken together they are one and true in the sense of the integrity that stands behind the very notion of Truth.

We divide and propagate dichotomies: system/observer, body/mind, object/process leading to object, self/other, and more. Only in the unity of such dichotomies are there classical truth values. In the sides of the division or distinction there are valid imaginary truth values exemplifying the strong non-locality of the Flagg Resolution. The world is one, and when it is broken into distinct parts these parts can partake of classical logic only in the non-local structure of the whole.

Does this sound like a discussion of non-locality in quantum physics? I believe that indeed it does. In splitting the world, in making a distinction, we can continue to use classical logic only in a context of non-local or global relations.

\section{THE RUSSELL PARADOX}

The Russell paradox shook the foundations of logic and mathematics at the turn of the century. It is simple and profound.

Let $\mathrm{AB}$ denote the statement that "B is a member of $\mathrm{A}$ ". With this notation we can define sets (collections) by equations. For example, the equation

$$
\mathbf{A x}=\mathbf{x x}
$$

states that "x is a member of A exactly when $\mathrm{x}$ is a member of itself" and the equation

$$
\operatorname{Rx}=\operatorname{Not}(\mathbf{x x})
$$

states that $\mathrm{x}$ is a member of $\mathrm{R}$ exactly when $\mathrm{x}$ is NOT a member of itself. $\mathrm{R}$ is the Russell set. 
The paradox occurs when we ask whether $\mathrm{R}$ can be a member of $\mathrm{R}$.

For if

$$
\operatorname{Rx}=\operatorname{Not}(\mathbf{x x})
$$

then, substituting $\mathrm{R}$ for $\mathrm{x}$, we have

$$
\mathbf{R R}=\operatorname{Not}(\mathbf{R R}) \text {. }
$$

$\mathrm{R}$ is a member of $\mathrm{R}$ exactly when $\mathrm{R}$ is not a member of $\mathrm{R}$.

We are in the domain of the Liar Paradox.

The Flagg Resolution takes care of the Russell paradox in exactly the same way it takes care of the Liar Paradox. The statement that

" $R$ is a member of $R$ exactly when $R$ is not a member of $R "$

is interpreted as a statement of substitution

$$
\begin{gathered}
\text { "'R is a member of } \mathrm{R} "=\text { " } \mathrm{R} \text { is not a member of } \mathrm{R} . " \\
\text { "RR = } \mathbf{N o t}(\mathbf{R R}) . "
\end{gathered}
$$

Note the implicit use of the equals sign as a transliteration of the words "exactly when". In fact it is by adopting these substitutions that we created the paradox in the first place. The Flagg resolution is an algebraic way get out of the train of difficulties engendered by a profligate use of the rule of substitution.

If you wish to replace R's self-membership by R's non-self membership anywhere then you must do so everywhere! It is not a local matter. The entire mathematical universe (or the universe delineated by a given text) waxes and wanes as a whole with respect to this statement. We can begin to see here the philosophical and mathematical import of the Resolution. The Resolution allows the Russell set to be a member of itself. The Resolution also allows the Russell set to NOT be a member of itself. The Resolution tells us that we must take these statements globally, as they are the roots (or factors) of a distinction.

Yes, concepts do define sets. The sets that concepts define bifurcate into sets that allow local substitution and behave in a "classical" manner, and sets like the Russell set that insist on an examination of the whole context before substitution is allowed. The Resolution forces us to examine the fundamental act of substitution in Mathematics and assures us, after examination, that Logic and Reason continue to function in these domains.

\section{Caveat Emptor}

There are other sets that partake of paradox. For example "the set of all sets that are not members of members of themselves" is also Russellian, but requires special analysis to reveal its deviltry.

More generally, the set of all well-founded sets is another devil, where we say that a set is well-founded if it contains no infinite descending chains of membership. These devils all give rise to a contradiction in the naive set theory, but the modes of revelation of the deception can be different in different cases. Flagg resolution is a method for dealing with known criminals. If you want to ensure that no criminals can live inside your walls, then there may be no recourse but to search them out with all available energy and then apply Flagg.

\section{CHARLES SANDERS PEIRCE AND THE SIGN OF ILLATION}

Charles Sanders Peirce wrote a remarkable essay [1] on the Boolean mathematics of a Sign that combines the properties of addition and negation. It is a portmanteau Sign in the sense of Lewis Carroll. See below for a rendition of it. 
Instead, I shall use the following version in this text: [a]b. When you see [a]b in the text you are to imagine that a horizontal bar has been placed over the top of the letter "a", and a vertical bar, crossed with a horizontal bar (very like a plus sign) has been placed just to the right of the "a" in such a way that the vertical bar and the horizontal overbar share a corner. In this way [a]b forms the Peirce Sign of illation, and we see that this Sign has been created by fusing a horizontal bar with a plus sign. The horizontal bar can be interpreted as negating the Sign beneath it.

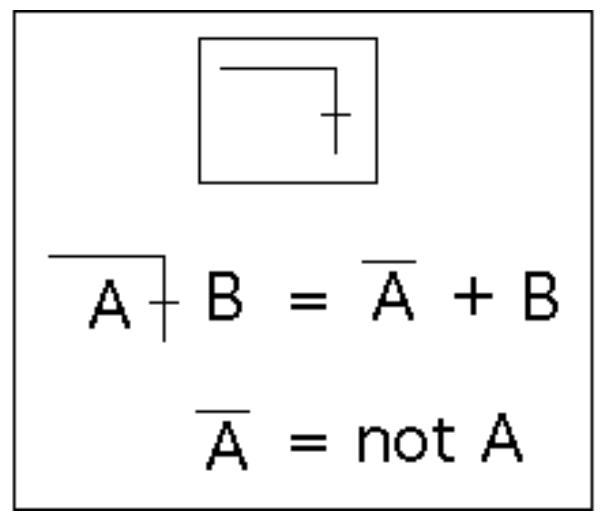

Peirce went on to write an essay on the formal properties of his Sign of illation and how it could be used in symbolic logic. Here is the dictionary definition of the word illation. Note that we have take the "Sign of Illation" as the title of this section of the paper.

il . la`tion. [L. illation, fr. illatus, used as past participle of inferre, to carry or bring in] Inference from premises of reasons; hence that which is inferred or deduced. [2]

The Sign of illation is a portmanteau Sign in the sense of Lewis Caroll [3], [4] who created that concept in his poem "Jabberwocky" where one encounters a bestiary of words like "slithy" - a combination of lithe and slimy. A portmanteau is literally a coat and hat rack (also a suitcase), an object designed to hold a multiplicity of objects. Just so, a portmanteau word is a holder of two or more words, each justly truncated to fit with the truncate of the other. A modern version to contemplate is the word "smog" a combination of "smoke" and "fog".

\section{PIVOT AND PORTMANTEAU}

Along with the concept of a portmanteau word or symbol there is a notion that I like to call a "pivot duality". A portmanteau word (The concept is due to Lewis Carroll.) is a combination of separate meanings such that their signs can be fitted together. In a pivot duality a word or symbol can be interpreted in more than one way, and this multiplicity of interpretation gives rise to a pivot, or translation, between the different contexts of these interpretations.

Pivot duality is the essence of multiplicity of interpretation, while portmanteau is the exemplar of the condensation of a multiplicity of meanings into a single sign. A portmanteau always has an associated pivot, but a pivot need not be a portmanteau. By bringing forth the pivot, we can expand the context of the consideration of the multiplicity of meanings associated with Signs. This has direct bearing on the understanding of the use of Signs in Peirce and in language as a whole.

A good example of pivot duality is the simple Feynman diagram

that can be interpreted (with time's arrow going up the page) as two particles interacting by the exchange of a photon (the dotted line), and can also be interpreted (with time's arrow going from left to right) as a particle and an antiparticle annihilating to produce a photon that then momentarily decays into a new particle pair. Here we have two completely different (yet related) interpretations of the same bit of formalism. The formalism seems to point to a deeper reality, beyond the particular way that the physicist observer decomposes process into space and time.

There is an affinity between the portmanteau symbol and a pivot. The portmanteau is a single word that holds two 
meanings. The pivot is a word or symbol or text that is subject to a multiplicity of interpretations. We make them both because these makings are the essence of the condensation of meaning into Signs and the use of Signs in the expansion of meaning. If the meaning of a Sign is its use, then the meaning of the Sign is not one but many, according to its uses, and yet one according to the unity that these uses find in the Sign itself (as a complex of Signs fully embedded in language). A hammer makes a good example, being one tool and yet being capable of both the impulsive insertion of the nail and the levering extraction of the nail. Two meanings pivot over the hammer. The combination of claw and hammerhead makes the tool itself a portmanteau of these two actions.

The reason, I believe, that portmanteau and pivot are so important to find in looking at formal systems, and in particular symbolic logic, is that the very attempt to make formal languages is fraught with the desire that each term shall have a single well assigned meaning. It cannot be! The single well-assigned meaning is against the nature of language itself. All the formal system can actually do is choose a line of development that calls some entities elementary (they are not) and builds other entities from them. Eventually meanings and full relationships to ordinary language emerge. The pattern of pivot and portmanteau is the clue to this robust nature of the formal language in relation to human thought and to the human as a Sign for itself.

The grin of the Cheshire cat is the quintessential pivot, yet it is not a portmanteau. To quote Martin Gardner in his comment on Alice's encounter with the Cheshire Cat [4, p. 91], “ The phrase 'grin without a cat' is not a bad description of pure mathematics. Although mathematical theorems can often be usefully applied to the structure of the external world, the theorems themselves are abstractions that belong in another realm 'remote from human passions,' as Bertrand Russell once put it in a memorable passage, 'remote even from the pitiful facts of Nature... an ordered cosmos where pure thought can dwell as in its natural home.' "

In mathematics the grin without the cat is often obtained through a process of distillation. The structure is traversed again and again and each time the inessential is thrown away. At last only a small and potent pattern remains. This is the grin of the cat. That grin is a pattern that fits into many contexts, a key to many doors. It is this multiplicity of uses for a single symbolic form that makes mathematics useful. It is the search for such distillation of pattern that is the essence of mathematical thought.

\section{LAWS OF FORM}

In Spencer-Brown's Laws of Form [9], the notation for an enclosure is not a circle, but a right angle bracket.

Thus the laws of calling and crossing as we have drawn them in circles become the following patterns in the right angle bracket:

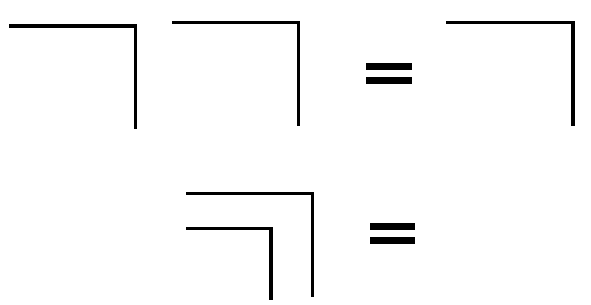

As we go from arithmetic to algebra and logic, Spencer-Brown makes the choice that AB (A juxtaposed with B) represents "A or B" rather than "A and B" as we have seen in the existential graphs and with the Sign of illation. However, with the marked state interpreted as "True" and the unmarked state interpreted as "False", implication in the Spencer-Brown algebra is given by the form shown below.

\section{$\mathrm{A} \mid \mathrm{B}=$ "A implies B"}

This puts implication in Laws of Form in exactly the same pattern as in Peirce's Sign of illation! 


\section{THE LOGICAL GARNET}

The purpose of this section is to point out a remarkable connection between Laws of Form, the Existential Graphs of Peirce, polyhedral geometry, mirror symmetry and the work of Shea Zellweger [30].

The details are in [42]. Here we will just exhibit the diagram representing Shea Zellweger's "logical garnet" and a few comments on it.

Zellweger did an extensive study of the sixteen binary connectives in Boolean logic ( "and", "or" and their relatives -- all the Boolean functions of two variables), starting from Peirce's own study of these patterns. He discovered a host of iconic notations for the connectives and a way to map them and their symmetries to the vertices of a four dimensional cube and to a three dimensional projection of that cube in the form of a rhombic dodecahedron. Symmetries of the connectives become, for Zellweger, mirror symmetries in planes perpendicular to the axes of the rhombic dodecahedron. See Figure 2. Zellweger uses his own iconic notations for the connectives to label the rhombic dodecahedron, which he calls the "Logical Garnet".

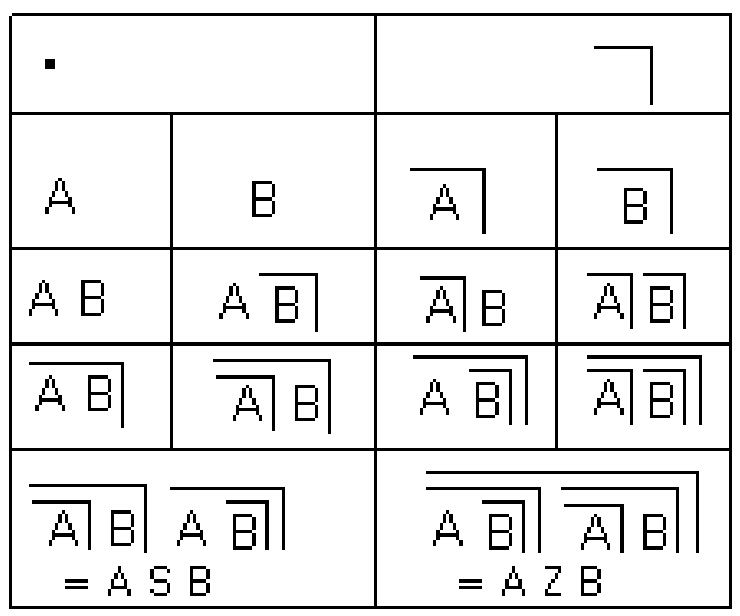

FIGURE 1. The Sixteen Binary Boolean Connectives

This is a remarkable connection of polyhedral geometry with basic logic. The meaning and application of this connection is yet to be fully appreciated. It is a significant linkage of domains. On the one hand, we have logic embedded in everyday speech. One does not expect to find direct connections of the structure of logical speech with the symmetries of Euclidean Geometry. It is the surprise of this connection that appeals to the intuition. Logic and reasoning are properties of language/mind in action. Geometry and symmetry are part of the mindset that would discover eternal forms and grasp the world as a whole. To find, by going to the source of logic, that we build simultaneously a world of reason and a world of geometry incites a vision of the full combination of the temporal and the eternal, a unification of action and contemplation. The relationship of logic and geometry demands a deep investigation. This investigation is in its infancy.

In this section we exhibit a version of the Logical Garnet (Figure 2) that is labeled so that each label is an explicit function of the two Boolean variables A and B. A list of these functions is given in Figure 1. We will find a new symmetry between the Marked and Unmarked states in this representation. In this new symmetry the mirror is a Looking Glass that has Peirce on one side and Spencer-Brown on the other!

View Figure 2. Note that in this three-dimensional figure of the Logical Garnet there are three planes across which one can make a reflection symmetry. Reflection in a horizontal plane has the effect of changing B to its crossed form in all expressions. Reflection in a vertical plane that is transverse to projection plane of the drawing, interchanges A and its crossed form. Finally, reflection in a plane parallel to the projection plane of the drawing interchanges marks with unmarks. Call this the Marked/Unmarked symmetry. 


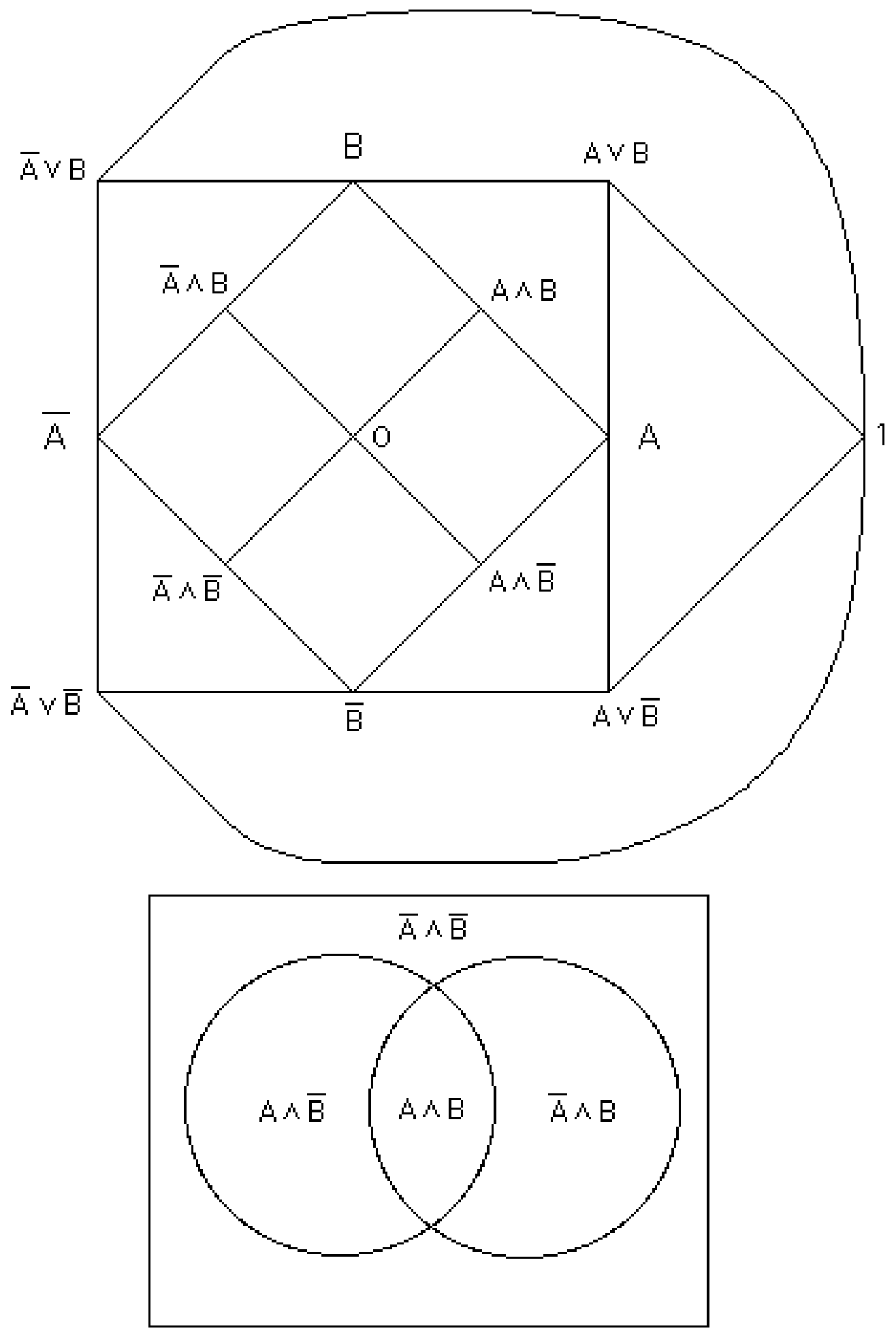

DIAGRAM 1. The Rhombic Dodecahedron as the Lattice Associated with a Two Circle Venn Diagram 


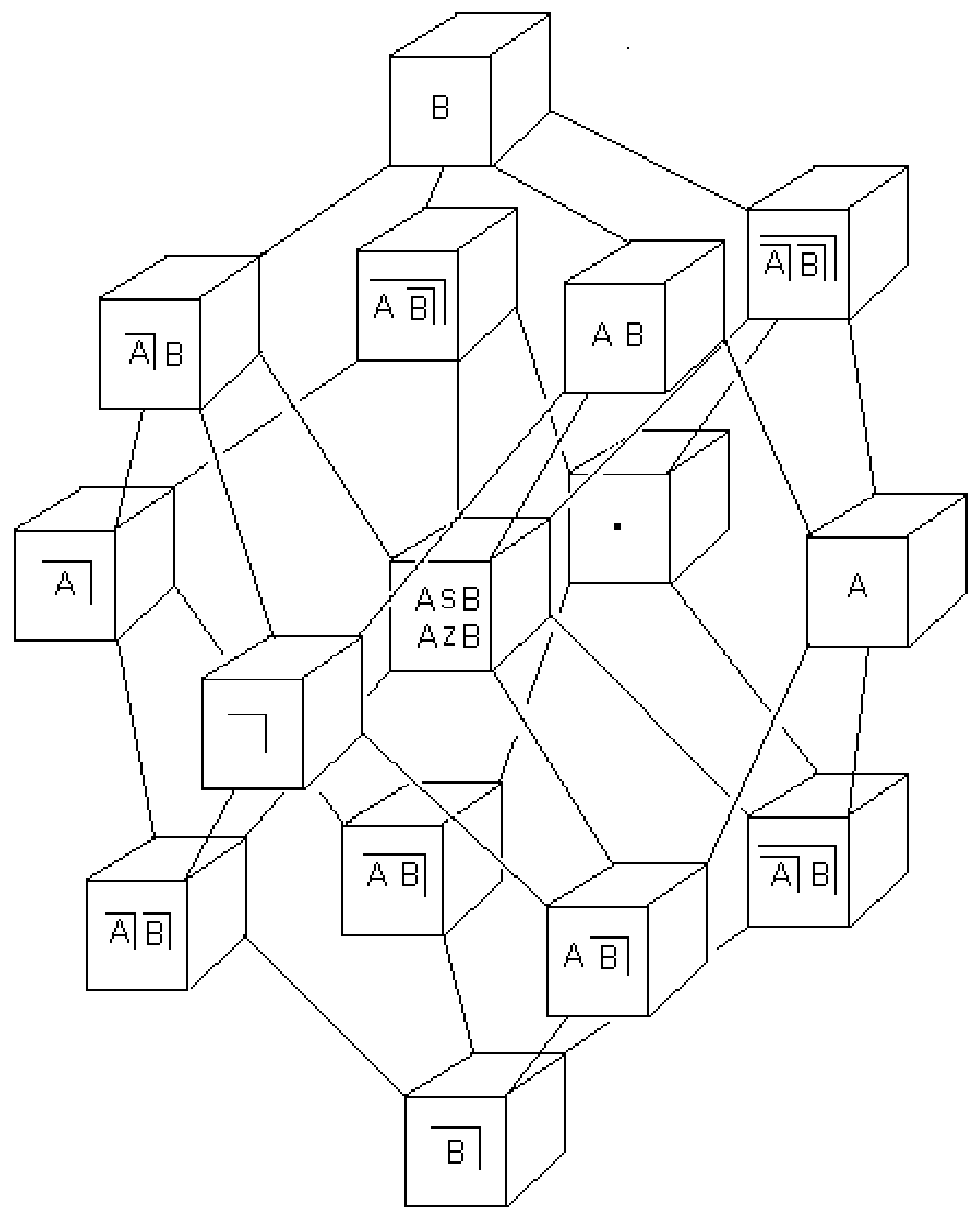

FIGURE 2 . The Logical Garnet

In this section we have exhibited a version of the Logical Garnet that intermediates between Peirce's Existential Graphs and the dual approach of Laws of Form. This appearance of significant Geometry at the very beginning of Logic deserves deeper investigation. The diagrammatic investigations of Peirce, Venn, Carroll, Nicod and SpencerBrown are all ways of finding geometry in logic, but in Zellweger's Logical Garnet classical three-dimensional geometry appears, and this is an indication that one should think again on the relationship of logic and mathematics. 


\section{PACKING MEANING}

We have seen that Logic can pack a double meaning, that Logic could be an encoded form of Geometry. Peirce's portmanteau Sign has expanded to a vastness of multiple interpretations. This was implicit in Boole's original symbolic logic. He borrowed the symbolism of ordinary algebra and invited that symbolism to carry the structure of class and inference. The fit of a good portmanteau operates like a key in a lock, opening a connection between the apparently separate domains that compose it.

Another portmanteau lives in Logic. It is the Gödelian sentence that asserts its own unprovability. This sentence carries a double meaning. Inside the formal system, it is a statement about properties of certain integers. Within the formal system there is no hint that the Gödel sentence has any other meaning. From outside the formal system, the sentence is seen to assert its own unprovability. These two meanings interlock in the compound Sign that is the Gödel sentence, to form a portmanteau that has forever changed our understanding of the nature of formal systems. This understanding is already present in Peirce through his view of the nature of the perceiving consciousness as a Sign for itself.

We ourselves are portmanteau Signs of a complex order. We are packing cases of multiple meaning large enough to make a human being a Sign of itself. As such we live in time, and this enables us to imagine timeless realms and realms of timeless synchronicity (such as the Flagg resolution) that allow the coexistence of clarity and paradox.

\section{REFERENCES}

1. C. S. Peirce, "The New Elements of Mathematics", edited by Carolyn Eisele, Volume IV - Mathematical Philosophy, Chapter VI - The Logical Algebra of Boole. pp. 106-115. Mouton Publishers, The Hague - Paris and Humanities Press, Atlantic Highlands, N. J. (1976).

2. Webster's New Collegiate Dictionary, G. C. Merriam Co. Pub. Springfield, Mass. (1956).

3. Lewis Carroll, “Alice's Adventures in Wonderland \& Through the Looking Glass”, (1865), (1871), (1988) Bantam Books.

4. Lewis Carroll with notes by Martin Gardner, “ The Annotated Alice - Alice's Adventures in Wonderland \& Through the Looking Glass" New American Library (1960).

5. J. G. P. Nicod, A Reduction in the number of Primitive Propositions of Logic. Proc. of Cambridge Phil. Soc. Vol. 19 (1916), pp. 32-40.

6. C. S. Peirce, "The New Elements of Mathematics", edited by Carolyn Eisele, Volume III/1 - Mathematical Miscellanea, Lowell Lectures ,1903. Lecture II, pp. 406-446. Mouton Publishers, The Hague - Paris and Humanities Press, Atlantic Highlands, N. J. (1976)

7. K. L. Ketner, "Elements of Logic - An Introduction to Peirce's Existential Graphs" Texas Tech University Press (1990).

8. C. S. Peirce, "Collected Papers - IV Chapter 3 - Existential Graphs", pp. 4.397-4.417, edited by Charles Hartshorne and Paul Weiss, Harvard University Press, Cambridge (1933).

9. G. Spencer-Brown, "Laws of Form", Julian Press, New York (1969).

10. F. J. Varela. Principles of Biological Autonomy. The North Holland Series in General Systems Research, G. Klir editor (1979). Elsevier North Holland Pub. (Chapter 12 - Closure and Dynamics of Forms).

11. H. R. Maturana and F. J. Varela, "The Tree of Knowledge - The Biological Roots of Human Understanding", New Science Library (1987).

12. L. H. Kauffman and F. J. Varela, Form dynamics, J. Soc. and Biological Structures (1984).

13. H. von Foerster. "Observing Systems", Objects: Tokens for Eigenbehaviours, pp. 274-285. Intersystems Publications (1981).

14. L. H. Kauffman. Self-reference and recursive forms. Journal of Social and Biological Structures (1987), 53-72.

15. L. H. Kauffman. The Robbins Problem - Computer Proofs and Human Proofs. (to appear in the Festschrift in honor of Gordon Pask).

16. L. H. Kauffman. Imaginary values in mathematical logic. Proceedings of the Seventeenth International Conference on Multiple Valued Logic, May 26-28 (1987), Boston MA, IEEE Computer Society Press, 282-289.

17. L. H. Kauffman, (1990). Robbins Algebra. Proceedings of the Twentieth International Symposium on Multiple Valued Logic. 54-60, IEE Computer Society Press.

18. L. H. Kauffman. Knot Logic. In Knots and Applications ed. by L. Kauffman, World Scientific Pub. (1994), pp. 1-110.

19. Huntington,E.V. (1933), Boolean Algebra. A Correction. Trans. Amer. Math. Soc. 35 , pp. 557-558.

20. A. Robinson, "Non-standard Analysis" (1966), North Holland - Amsterdam.

21. J. H. Conway, "On Numbers and Games" Academic Press (1976).

22. J. L. Bell, “A Primer of Infinitesimal Analysis”, (1998) Cambridge University Press.

23. J. M. Henle, Non-nonstandard analysis: real infinitesimals, Mathematical Intelligencer, Vol. 21, No 1. (1999), pp. 67-73. Springer-Verlag, New York.

24. C. S. Peirce, "Collected Papers - II, p. 2.230-2.231, edited by Charles Hartshorne and Paul Weiss, Harvard University Press, Cambridge (1933). 
25. H. P. Barendregt. "The Lambda Calculus Its Syntax and Semantics", North Holland (1981 and 1985).

26. R. Courant and H. Robbins, "What is Mathematics?", Oxford University Press (1941 and 1969).

27. F. W. Lawvere, Adjointness in foundations, Dialectica 23:82 (1969).

28. D. Scott, Continuous lattices, in "Toposes Algebraic Geometry and Logic", edited by F. W. Lawvere, pp. 97-136. Springer Verlag Lecture Notes in Mathematics Vol. 274 (1970).

29. S. Maclane and 1. Moerdijk, "Sheaves in Geometry and Logic", Springer-Verlag (1992).

30. S. Zellweger, Untapped potential in Peirce's iconic notation for the sixteen binary connectives, in "Studies in the Logic of Charles Peirce", edited by N. Hauser, D.D. Roberts and J. V. Evra, Indiana University Press (1997), pp. 334-386.

31. D. Hofstadter, "Godel, Escher, Bach: An Eternal Golden Braid" Basic Books Inc. (1979).

32. E. Nagel and J. R. Newman, "Godel's Proof", New York University Press (1960).

33. J. N. Crossley et al, "What is Mathematical Logic?", Oxford University Press (1972).

34. R. Robertson, One, Two Three ... Continuity: C. S. Peirce and the Continuum, Cybernetics and Human Knowing (2001).

35. L. H. Kauffman, "Virtual Logic", columns in Cybernetics and Human Knowing.

36. L.H. Kauffman and J. M. Flagg. The Flagg Resolution. (in preparation).

37. L. H. Kauffman. DeMorgan Algebras - Completeness and Recursion. Proceedings of the Eighth International Conference on Multiple Valued Logic(1978),. IEEE Computer Society Press, 82-86.

38. S. C. Kleene. Introduction to Metamathematics. P. Noordhof New York, Groningen (1952).

39. J. M. Flagg. (Private Communication - 1999).

40. F. J. Varela. A calculus for self-reference. (1975). Int. J. Gen. Systems.

41. L. Wittgenstein. Lectures on the Foundations of Mathematics. edited by Cora Diamond, Cornell Univ. Press (1976). Lecture XXI, p. 211.

42. L. H. Kauffman, The mathematics of Charles Sanders Peirce. Cybernetics and Human Knowing, Vol. 8, No. 1-2, (2001), 79110.

43. L. H. Kauffman. Knot Logic. In "Knots and Application" edited by L. H. Kauffman, World Scientific Pub. Co. (1994), pp. 1110

44. L.H.Kauffman and H. C. Sabelli. The Process Equation. Cybernetics and Systems. Vol. 29 (1998), pp. 345-362. 